\title{
Bewegungsdeterminanten, Erfüllung der Empfehlungen für ausdauerorientierte Bewegung und Gesundheit: Ergebnisse einer Korrelationsstudie aus den österreichischen Bundesländern
}

\author{
Determinants of Exercise, Fulfilling the Recommendations for \\ Aerobic Physical Activity and Health Status: Results of a \\ Correlation Study in the Federal States of Austria
}

\section{(우요 $\odot$}

\author{
Autoren \\ Thomas Ernst Dorner ${ }^{1,2}$, Sandra Haider ${ }^{1}$, Christian Lackinger ${ }^{1}$, Ali Kapan ${ }^{1}$, Sylvia Titze ${ }^{3}$
}

Institute

1 Abteilung für Sozial- und Präventivmedizin, Zentrum für Public Health, Medizinische Universität Wien, Austria

2 Sozialversicherung öffentlich Bediensteter, Eisenbahnen und Bergbau, Wien, Austria

3 Institut für Sportwissenschaft, Universität Graz, Graz, Austria

\section{Schlüsselwörter}

Korrelation, Bundesländer, Kultur, Determinanten,

Gesundheitszustand, Wissen

\section{Key words}

Correlation, federal states, culture, determinants, health status, knowledge

\section{Bibliografie}

Gesundheitswesen 2020; 82 (Suppl. 3): S207-S216

DOI 10.1055/a-1191-4309

ISSN 0949-7013

(c) 2020. The Author(s).

This is an open access article published by Thieme under the terms of the Creative Commons Attribution-NonDerivative-NonCommercial-License, permitting copying and reproduction so long as the original work is given appropriate credit. Contents may not be used for commercial purposes, or adapted, remixed, transformed or built upon. (https://creativecommons. org/licenses/by-nc-nd/4.0/)

\section{Korrespondenzadresse}

Prof. Thomas Ernst Dorner

Abteilung für Sozial- und Präventivmedizin

Zentrum für Public Health

Medizinische Universität Wien

Kinderspitalgasse 15/I

1090 Wien, Austria

thomas.dorner@meduniwien.ac.at

\section{ZUSAMMENFASSUNG}

Ziel der Studie Ziel dieser Analyse war es, Zusammenhänge zu untersuchen zwischen Personen, die die ausdauerorientierten Bewegungsempfehlungen erfüllen und dem Wissen um die Bewegungsempfehlungen, die wahrgenommene bewegungsförderliche Kultur und Umgebung sowie den Gesundheitszustand getrennt nach Bundesländern.

Methodik Für die Analyse wurden Daten aus dem österreichischen Bewegungsmonitoring 2017, der österreichischen Gesundheitsbefragung 2014, der Mortalitätsstatistik 2015, der Fit-Sport-Austria Datenbank, der Wirtschaftskammer, dem Verkehrsministerium und einer Online-Lauf-Plattform herangezogen. Korrelationskoeffizienten (Pearson) verschiedener Parameter mit den Bevölkerungsanteilen, die die ausdauerorientierten Bewegungsempfehlungen erfüllten, wurden errechnet.

Ergebnisse Ausdauerorientierte Bewegung korrelierte signifikant mit dem Wissen zum empfohlenen Bewegungsumfang $(0,91)$, Angeboten in $(0,87)$ und subjektiver Erreichbarkeit von Sportvereinen $(0,85)$, Erreichbarkeit von Grünräumen $(0,84)$, Anzahl an Sportvereinen mit zertifizierten gesundheitsförderlichen Angeboten $(0,76)$, und den Möglichkeiten Laufen zu gehen $(0,72)$. Außerdem korrelierte ausdauerorientierte Bewegung negativ mit der altersstandardisierten kardiovaskulären Mortalität $(-0,96)$, der Gesamtmortalität $(-0,95)$, der Prävalenz von Adipositas $(-0,83)$, der Mortalität an ischämischen Herzerkrankungen $(-0,76)$, der Prävalenz chronischer Krankheiten $(-0,68)$, sowie positiv mit der subjektiv wahrgenommenen Gesundheit $(0,79)$.

Schlussfolgerung Zum einen zeigten sich positive signifikante Zusammenhänge zwischen Wissen um die Bewegungsempfehlungen, der Infrastruktur und der bewegungsförderlichen Kultur mit dem Bewegungsverhalten. Zum anderen korrelierte das Bewegungsverhalten mit dem Gesundheitszustand in den jeweiligen Bundesländern. Zusätzlich lassen sich spezifische Empfehlungen, zugeschnitten auf die einzelnen Bundesländer, ableiten. 


\section{ABSTRACT}

Study aim The aim of this study was to examine the association between the proportion of the population that fulfilled the endurance-based recommendations for health-enhancing physical activity and the proportion with correct knowledge of these recommendations, exercise-friendly culture and environment, and health status in the federal states of Austria.

Methods The analysis is based on data from the Austrian Physical Activity Monitoring 2017, the Austrian Health Interview Survey 2014, the Austrian Mortality Register 2015, the database of Fit Sports Austria, the Austrian Chamber of Commerce, the Federal Ministry for Traffic, and an online platform for running events. Correlation coefficients (Pearson) for the various parameters and the proportion of the population that fulfilled the endurance-based recommendations for health-enhancing physical activity are presented.

Results There is a significant correlation between endurancebased physical activity and the knowledge regarding the re- commended extent of physical activity (0.91), the number of offers in sports clubs (0.87), the subjective accessibility of sports clubs (0.85), the accessibility of green spaces (0.84), the number of sports clubs with certified health-promoting programs (0.76), and the perceived possibilities to go running (0.72). In addition, endurance-based physical activity is significantly and negatively correlated with age-adjusted cardiovascular mortality $(-0.96)$, total mortality $(-0.95)$, the prevalence of obesity $(-0.83)$, ischemic heart mortality $(-0.76)$, and the prevalence of chronic diseases in total $(-0.68)$, and is positively correlated with subjective health (0.79).

Conclusions The correlations show the significant associations between exercise behavior and health knowledge, the infrastructure for exercise, and culture. In addition, they show the significant correlation between exercise behavior and health status in the Austrian federal states. This information is important for tailored recommendations for the individual federal states.

\section{Einleitung}

Die Effekte regelmäßiger körperlicher Aktivität auf die physische Gesundheit, das psychische und soziale Wohlbefinden, auf die Ökonomie und die Umwelt sind wissenschaftlich überzeugend nachgewiesen [1-3]. Um Auskunft zu geben welche Art, Intensität, Dauer und Häufigkeit von Bewegung nötig ist, werden internationale und nationale Bewegungsempfehlungen erstellt. In Bezug auf ausdauerorientierte Bewegung lauten die aktuellen österreichischen Bewegungsempfehlungen aus dem Jahr 2020 für die erwachsene Bevölkerung, dass „Erwachsene mindestens 150 Min. (21⁄2 Stunden) bis 300 Min. (5 Stunden) pro Woche ausdauerorientierte Bewegung mit mittlerer Intensität oder 75 Min. (1 1/4 Stunden) bis 150 Min. (2 1/2 Stunden) pro Woche ausdauerorientierte Bewegung mit höherer Intensität oder eine entsprechende Kombination aus ausdauerorientierter Bewegung mit mittlerer und höherer Intensität durchführen" sollen [4].

Damit ein möglichst hoher Anteil der Bevölkerung diese Empfehlungen auch erfüllt, braucht es gesellschaftlicher und persönlicher Voraussetzungen. Basierend auf dem sozial-ökologischen Modell [5], bei dem auf verschiedenen Ebenen Einflussfaktoren auf die Gesundheit dargestellt wurden, können verschiedene Einflussfaktoren auf das individuelle Bewegungsverhalten definiert werden. Diese Einflussfaktoren auf vier Ebenen sind folgende [2]:

1. Individuelle Verhaltens- und Lebensweise: Einstellung, Motivation, Willensbildung, Erwartungen, motorische Fähigkeiten und Fertigkeiten.

2. Soziales Umfeld und Netzwerk: Partnerschaft, Familie, Freunde, Peergroups, Vereine, Netzwerke, ethnische Zugehörigkeit, Religion.

3. Lebens- und Arbeitsbedingungen: Schule, Arbeit, Mobilität, Freizeit, Freizeit- und Sportindustrie, Gesundheitsdienste, Sozialversicherung.

4. Gebaute Umwelt, sozioökonomisches und politisches Umfeld: Wohnverhältnisse, Wohnregion, Infrastruktur (öffentlich, kommer- ziell, naturnah), Wirtschaftslage, Einkommen, Medien, Bundessportorganisation, Fonds Gesundes Österreich und Ministerien.

Auf Bevölkerungsebene sind für mehr Bewegung v. a. 3 Parameter wichtig: Es braucht eine entsprechende Kommunikation der Bewegungsempfehlungen an die Bevölkerung, um das Wissen über die Bedeutung und das empfohlene Ausmaß gesundheitswirksamer Bewegung in der Bevölkerung zu verbreiten. Weiter braucht es eine bewegungsförderliche Kultur, in der es als „normal“ und „sozial erwünscht" angesehen wird, Bewegung im Sinne der Empfehlungen zu machen. Solch eine Kultur wird durch bewegungsfördernde Angebote (Veranstaltungen, Infrastruktur, Vereine, gebaute Umwelt die zu Bewegung animiert, Grünflächen, gesetzliche Rahmenbedingungen usw.) generiert, die als dritte wesentliche Voraussetzung für mehr Bewegung gesehen werden. Der dabei hypothetisierte Zusammenhang wird in > Abb. 1 dargestellt. Die 3 Faktoren (1) bewegungsförderliche Kultur, (2) Wissen in der Bevölkerung und (3) bewegungsförderliche Umgebung stehen untereinander auch in Beziehung. So hat der Stellenwert von Bewegung in der Gesellschaft auch einen Einfluss darauf, wie das Wissen zu Gesundheitswirkungen körperlicher Aktivität in der Gesellschaft ist. Zusätzlich bedingt der Stellenwert von Bewegung in der Gesellschaft auch die bewegungsfördernde Umgebung; je höher der Stellenwert, umso mehr Angebote für Bewegung werden zur Verfügung gestellt werden. Dieser Zusammenhang ist aber reziprok, da der Stellenwert von Bewegung in der Gesellschaft auch von der bewegungsförderlichen Umgebung abhängt; je besser die Infrastruktur, und je sichtbarer dadurch Bewegung in der Gesellschaft ist, umso mehr wird der Stellenwert von Bewegung in der Gesellschaft steigen.

In diesem Modell ist auch der Zusammenhang zwischen dem Bewegungsverhalten und dem Gesundheitszustand der Bevölkerung dargestellt. Der Gesundheitszustand folgt dem Ausmaß von Bewegung in der Bevölkerung, allerdings zeitversetzt. Steigt das Bewegungsausmaß in der Bevölkerung, wird sich rasch die subjektive Gesundheit erhöhen, später wird die Prävalenz von chronischen Krankheiten, wie Adipositas, Bluthochdruck und Diabetes mellitus 


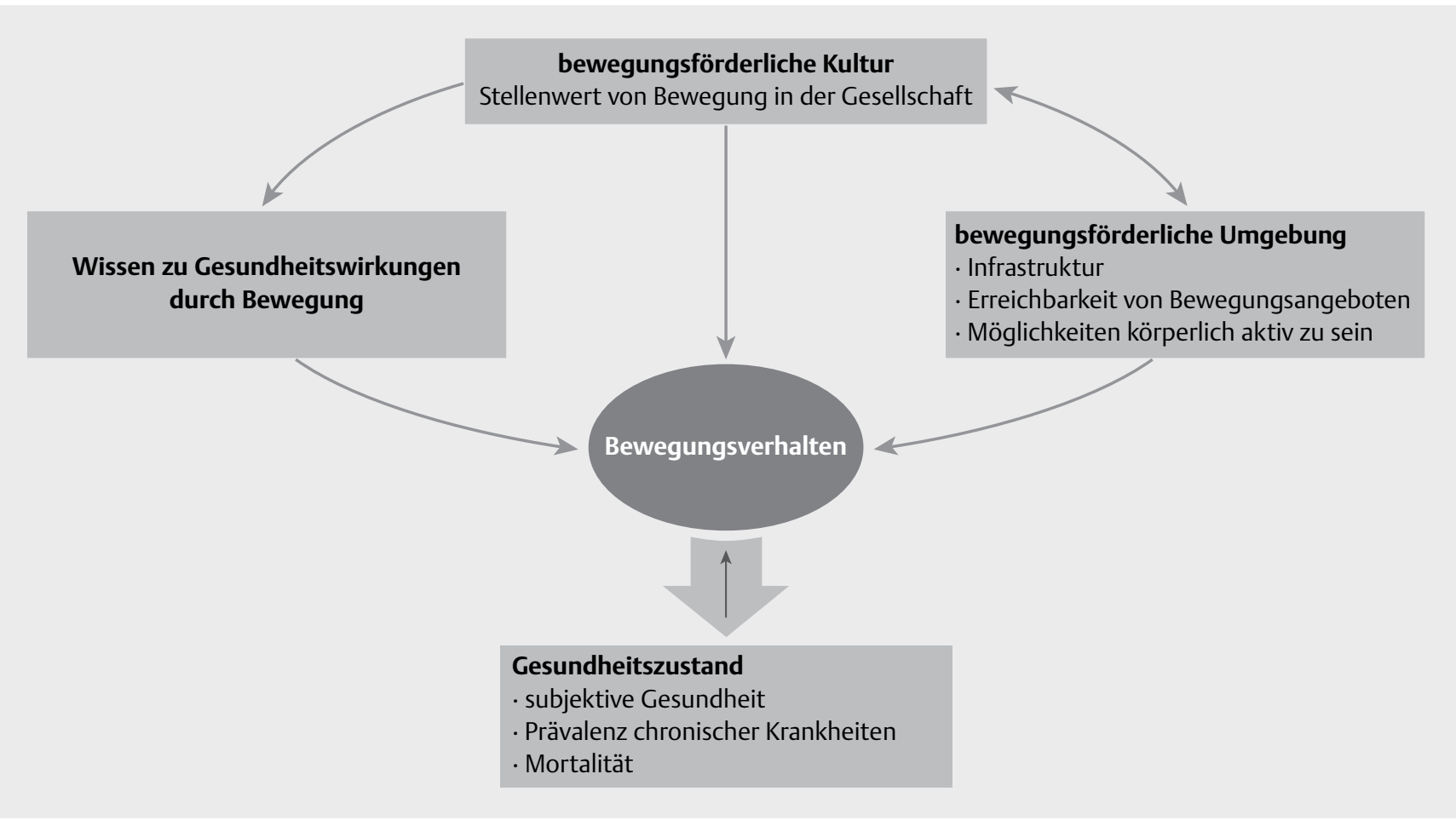

Abb. 1 Einflussfaktoren auf das Bewegungsverhalten und Zusammenhang mit dem Gesundheitszustand einer Bevölkerung.

sinken, und noch später wird die Mortalität sinken. Dabei ist jedoch auch zu berücksichtigen, dass in geringerem Ausmaß der Gesundheitszustand einer Bevölkerung auch das Bewegungsverhalten determiniert. Körperliche Beeinträchtigungen können Bewegung erschweren und den Bewegungsumfang reduzieren.

Österreich ist ein Land mit neun Bundesländern, in dem Föderalismus, aber auch Zentralismus eine große Bedeutung beigemessen wird. Es gibt nationale Rahmenprogramme für Gesundheit, wie die österreichischen Gesundheitsziele, in dem auch Bewegung adressiert wird, und für Sport, wie den nationalen Aktionsplan Bewegung [6]. Die Förderung von Sport in Vereinen, die Etablierung bewegungsförderlichen Umweltbedingungen und die gesundheitliche Versorgung inklusive Gesundheitsmonitoring sind in Österreich aber zu großem Teil Aufgaben der einzelnen Bundesländer [7].

Um den Zusammenhang zwischen Bewegung, der sie determinierenden Faktoren und Gesundheit in Österreich besser verstehen zu können, um zu messen, wie sich das Bewegungsverhalten auf Bevölkerungsebene entwickelt, und um Interventionen für mehr Bewegung evaluieren zu können, wurde ein Konzept für ein Bewegungsmonitoring-System in Österreich entwickelt [8]. Von Juli bis November 2017 wurde eine Basis-Befragung durchgeführt und publiziert [9]. Mit diesem Monitoring-System werden existierende Erhebungsinstrumente im Bereich Bewegung und Gesundheit, wie die Österreichische Gesundheitsbefragung, ergänzt [10].

Vor diesem Hintergrund war es die Absicht dieser Analyse, das ausdauerorientierte Bewegungsverhalten basierend auf den vorhandenen Datensätzen für die Österreichischen Bundesländer zu analysieren und zu erheben, in welchem Ausmaß dieses mit bewegungsdeterminierenden Faktoren, aber auch mit dem Gesundheitszustand in den einzelnen Bundesländern in Zusammenhang steht.

\section{Methodik}

Bei allen Daten, die in diesem Bericht verwendet werden, handelt es sich um auf Bundeslandebene aggregierte Daten, und keine Individualdaten. Das heißt, es wurden die Merkmale von nur neun Beobachtungseinheiten (Bundesländer) analysiert. Für die Analyse wurden Daten aus 2 Befragungen herangezogen: dem österreichischen Bewegungsmonitoring 2017 und der österreichischen Gesundheitsbefragung 2014. Weiter wurden Daten zu bewegungsförderlicher Infrastruktur aus verschiedenen Datenquellen verwendet und diese auf die Bevölkerung in den einzelnen Bundesländern bezogen. Zusätzlich wurden Daten aus der aktuellsten zur Verfügung stehenden österreichischen Mortalitätsstatistik (aus dem Jahr 2015) herangezogen.

\section{Österreichisches Bewegungsmonitoring 2017}

Das Österreichische Bewegungsmonitoring ist ein Datensatz, der von Juli bis November 2017 im Auftrag des Bundesministeriums für Landesverteidigung und Sport vom Institut für empirische Sozialforschung erhoben wurde. Der verwendete Fragebogen wurde von der Kompetenzgruppe Körperliche Aktivität / Bewegung / Sport der Österreichischen Gesellschaft für Public Health im Auftrag des Sportministeriums erstellt. Der Stichprobenumfang betrug 4000 Personen ab 15 Jahren, die in Bezug auf Geschlecht und groben Altersstrukturen repräsentativ für die österreichische Bevölkerung und die Bevölkerung in den einzelnen Bundesländern ist. Die Daten wurden durch eine mündliche persönliche Befragung (Computer assisted personal Interviews, CAPI) an der Wohnadresse erhoben. Gefragt wurde nach dem Ausmaß der Bewegung, Wissen um den zeitlichen Umfang und die Intensität von Bewegung um Gesundheitseffekte zu erreichen, Einschätzung der eigenen Aktivitäten, 
Stellenwert von Bewegung in der Gesellschaft, Infrastruktur und Bewegungsangebote, sowie Gesundheitszustand und Lebensqualität [9]. Für die Analyse wurden die Daten gewichtet, um eine höhere Repräsentativität zu erzielen.

Bei diesem Datensatz kam der Global Physical Activity Questionnaire (GPAQ) zum Einsatz, ein Fragebogen mit ausreichender Reliabilität und Validität $[11,12]$. Die Berechnung des Anteils der Personen, die die ausdauerorientierten Bewegungsempfehlungen erfüllte, erfolgte folgendermaßen: Die Antwort auf die Frage „Betreiben Sie in der Freizeit zumindest einmal in der Woche intensive körperliche bzw. sportliche Aktivitäten, bei denen Atmung und Puls für durchgehend zumindest 10 Min. stark zunehmen, wie z. B. beim Laufen oder Fußballspielen? An wie vielen Tagen einer gewöhnlichen Woche machen Sie das in der Freizeit? “ wurde mit der angegebenen mittleren Dauer pro Tag in Minuten und zusätzlich mit dem Faktor 2 multipliziert (da als Faustregel 10 Min. Bewegung mit höherer Intensität 20 Min. Bewegung mit mittlerer Intensität ersetzen können). Dazu wurde die Dauer der Bewegung mit mittlerer Intensität pro Woche addiert ( = Ergebnis der Multiplikation der Antwort auf die Frage „An wie vielen Tagen einer gewöhnlichen Woche betreiben Sie in der Freizeit körperliche Aktivitäten oder Sport mit mittlerer Intensität?“ mit der Antwort auf „Und für wie lange ca. betreiben Sie an einem solchen Tag in der Freizeit körperliche Aktivitäten oder Sport mit mittlerer Intensität?"). Lag die Summe aus doppelt gezählten Minuten pro Woche aus Bewegung in der Freizeit mit höherer Intensität und den einfach gezählten Minuten pro Woche aus Bewegung in der Freizeit mit mittlerer Intensität den Wert bei oder über 150 Min. Bewegung pro Woche, wurde definiert, dass die ausdauerorientierten Bewegungsempfehlungen erfüllt wurden.

Zusätzlich wurden Fragen zum Thema Bewegungskompetenz gestellt, welche aus dem Schweizerischen Monitoring-System Ernährung und Bewegung (MOSEB) entnommen wurden [13]. Eine Frage zum Stellenwert von Bewegung in unserer Kultur wurde in Anlehnung an das Österreichische Jugendportal Bewegung und Sport gestellt [14]. Fragen zur bewegungsförderlichen Infrastruktur wurden aus dem Eurobarometer der Europäischen Kommission [15] sowie aus einem Fragebogen des Projektes „Neighborhood Environment Walkability Scale“ entnommen [16].

\section{Österreichische Gesundheitsbefragung (ATHIS) 2014}

Die Österreichische Gesundheitsbefragung (Austrian Health Interview Survey, ATHIS) ist eine Studie, die regelmäßig von der Statistik Austria in der österreichischen Allgemeinbevölkerung ab 15 Jahren durchgeführt wird. Für diesen Bericht wurde die 2. Welle im Jahr 2014 herangezogen. ATHIS basiert auf dem European Health Interview Survey (EHIS) $[17,18]$, der in 17 europäischen Ländern mit einem standardisierten Kernfragebogen auf die gleiche Weise durchgeführt wird.

Der ATHIS 2014 wurde von Oktober 2013 bis Juni 2015 größtenteils mittels Telefoninterviews (computer assisted telephone interview, CATI) erhoben. Einige Fragen, so auch die Fragen zu körperlicher Aktivität wurden via Paper-Pencil-Fragebogen erhoben und die Fragebögen postalisch retourniert. Insgesamt wurden 38769 Personen im Rahmen der Mikrozensus-Erhebungen durch Statistik Austria eingeladen, beim ATHIS mitzumachen. Im Endeffekt konnten die Daten von 15771 Personen analysiert werden, was einer Antwortrate von $41 \%$ entspricht. Die Responserate für den Papier-Fragebogen betrug 93\%. Um die Repräsentativität zu erhöhen, wurden die Daten für die Analyse nach geografischer Region, Alter, Geschlecht, familiärer Situation, Migrationshintergrund und Ausbildungsstatus gewichtet [10].

Der ATHIS enthält Fragen zum Gesundheitszustand, Gesundheitsversorgung, sowie gesundheitlichen Einflussfaktoren und gesundheitsrelevantem Lebensstil. Um die körperliche Aktivität zu erheben wurde der Physical Activity Questionnaire des EHIS (EHISPAQ) verwendet [19]. Dieser basiert auf den GPAQ, und besteht aus acht Fragen zu Bewegung während der Arbeit, beim Transport und in der Freizeit in einer typischen Woche, wobei nur Tätigkeiten, die länger als 10 Min. am Stück dauern, berücksichtigt werden. Außerdem wird nach muskelkräftigenden Übungen gefragt. Der EHISPAQ zeigt gute Werte für Reliabilität und Validität [20]. Bezüglich körperlicher Aktivität wurde im ATHIS folgende Eingangsfrage gestellt: „Wir möchten gerne wissen, wie viel Zeit Sie in einer typischen Woche mit der Ausübung verschiedener körperlicher Aktivitäten verbringen. Bitte beantworten Sie die Fragen auch dann, wenn Sie sich selbst nicht für eine körperlich aktive Person halten. Denken Sie zuerst an die Zeit, in der Sie arbeiten, also an Dinge, die Sie tun müssen, wie beispielsweise bezahlte und unbezahlte Arbeit, Arbeiten rund um Ihr Zuhause, Kinderbetreuung, Pflege von Angehörigen, Schule oder Studium“. Weiter wurde die Frage gestellt: „Schließen Sie bei den nächsten Fragen die arbeitsbezogenen körperlichen Aktivitäten aus, die Sie bereits genannt haben. Wir möchten gerne wissen, wie Sie sich üblicherweise von A nach B fortbewegen. Zum Beispiel zur Arbeit, zur Universität, zur Schule oder zum Einkaufen. An wie vielen Tagen in einer typischen Woche fahren Sie mindestens 10 Min. ohne Unterbrechung mit dem Fahrrad, um von A nach B zu gelangen?“, und „Wie lange fahren Sie an einem typischen Tag mit dem Fahrrad, um von A nach B zu gelangen?“ Als nächstes wurde gefragt: „Schließen Sie bei der nächsten Frage die körperliche Aktivität bei der Arbeit und um von A nach B zu gelangen aus, die Sie bereits genannt haben. In den folgenden Fragen geht es um Sport, Fitness und körperliche Aktivität in der Freizeit, welche mindestens zu einem leichten Anstieg der Atem- oder Herzfrequenz führen. Zum Beispiel (Nordic-) Walking, Ballsport, Joggen, Fahrradfahren, Schwimmen, Aerobic, Rudern oder Badminton. An wie vielen Tagen in einer typischen Woche üben Sie mindestens 10 Min. ohne Unterbrechung Sport, Fitness oder körperliche Aktivität in der Freizeit aus?“, und schließlich „Wie viel Zeit verbringen Sie insgesamt in einer typischen Woche mit Sport, Fitness oder körperlicher Aktivität in der Freizeit?". Die Minuten pro Woche, die mit Radfahren und mit Sport, Fitness und körperlicher Aktivität in der Freizeit verbrachten wurden, wurden addiert. Hat nun diese Summe den Wert 150 erreicht oder überschritten, wurde definiert, dass die Bewegungsempfehlungen für Ausdauerbewegung erfüllt wurden.

Außer den Fragen zu Bewegung wurden aus dem ATHIS folgende Parameter entnommen: Prävalenz chronischer Krankheiten, Body Mass Index (BMI) berechnet mit selbstberichteten Daten zu Körpergewicht und Körpergröße, Prävalenz von Bluthochdruck und Diabetes mellitus sowie die subjektiv wahrgenommene Gesundheit.

\section{Indikatoren zu bewegungsförderlicher Infrastruktur}

Für die bewegungsförderliche Infrastruktur wurden verschiedene Datenquellen herangezogen. Dazu zählen Publikationen der Bun- 
dessportorganisation, der Wirtschaftskammer Österreich und des Verkehrsministeriums. Es konnten nur Daten verwendet werden, bei denen vergleichbare Zahlen für alle Bundesländer vorlagen. Die folgenden Indikatoren für bewegungsförderliche Infrastruktur wurden für den vorliegenden Bericht herangezogen:

Vereine mit zertifizierten gesundheitsfördernden Angeboten für Erwachsene und Anzahl der gesundheitsfördernden Angebote für Erwachsene in Vereinen wurden aus der Fit Sport Austria Datenbank entnommen. Dabei wurden ausschließlich Sportvereine bzw. Bewegungsangebote, die von der Fit Sport Austria $\mathrm{GmbH}$ (FSA) mit einem Qualitätssigel zertifiziert wurden, herangezogen. Dieses Qualitätssiegel wird dann vergeben, wenn folgende drei Kriterien erfüllt sind: qualifizierte Übungsleiterinnen und Übungsleiter, qualifiziertes Angebot, und Qualitätssicherung. Die Abfrage erfolgte am 10. Juli 2019 [21]. Die Anzahl an Vereinen mit gesundheitsfördernden FSA zertifizierten Angeboten und die Anzahl dieser gesundheitsfördernden Angebote wurden für diesen Bericht auf die Bevölkerung in den jeweiligen Bundesländern bezogen. Bevölkerungszahlen wurden den Publikationen von der Statistik Austria entnommen [22].

Gewerbliche Sportanbieter wurden über die Wirtschaftskammer Österreich (WKO) erfasst. Die Aufzeichnung über den Stand der einzelnen Mitglieder bzw. Mitgliedsbetriebe basiert auf Daten der einzelnen Bundesländer. Für die Analyse in diesem Bericht wurden Fitnessbetriebe entsprechend der Codierung 0300 „Fitnessbetriebe (Fitnessstudios, Fitnesscenter) “ aus dem Jahr 2018 herangezogen [23]. Die Anzahl an Fitnessbetrieben wurde für diesen Bericht auf die Bevölkerung in den jeweiligen Bundesländern bezogen. Bevölkerungszahlen wurden den Publikationen von der Statistik Austria entnommen [22].

Zahlen zur Infrastruktur und zum Radverkehr wurden aus einer Publikation des österreichischen Verkehrsministeriums entnommen. Dazu wurde die Anzahl an Fahrrädern pro 1000 Einwohnerinnen und Einwohner sowie die Radwegenetzlänge pro km² in Meter herangezogen [24].

Die Startfelder bei Straßenläuferinnen und bei Straßenläufern bestehen zum überwiegenden Anteil aus Hobby- und Amateursportlerinnen und -sportler. Somit ist die Anzahl der Laufveranstaltungen in einem Bundesland pro Jahr ein guter Indikator für bewegungsförderliche Infrastruktur. Für die Analyse wurde die Online-Plattform www.laufkalender24.at herangezogen. Die Vollständigkeit der Termine wurde von mehreren Läuferinnen und Läufern aus dem Hobbyund Amateurbereich überprüft. Im Menü „für Läufer“ können unter „Lauf suchen“ die Laufveranstaltungen eines Bundeslandes abgerufen werden. Die Anzahl an Laufveranstaltungen für das Jahr 2019 wurde für diesen Bericht herangezogen. Die Anzahl an Laufveranstaltungen wurde für diesen Bericht auf die Bevölkerung in den jeweiligen Bundesländern bezogen. Bevölkerungszahlen wurden den Publikationen von der Statistik Austria entnommen [22].

\section{Mortalitätsregister}

In der Todesursachenstatistik wird in Österreich jeder Todesfall dokumentiert. Die Todesursachen werden von einer Totenbeschauärztin / einem Totenbeschauarzt, Pathologin / Pathologen oder Gerichtsmedizinerin / Gerichtsmediziner angegeben und nach ICD (International Classification of Diseases) kodiert [19]. Für diesen Bericht wurde die Gesamtmortalität (Gestorbene pro 100000
Einwohner/innen), die Mortalität an Herz- Kreislauferkrankungen (Verstorbene an Krankheiten, die nach ICD-10 mit 100 bis 199 „Krankheiten des Kreislaufsystems“ kodiert wurden pro 100000 Einwohner/innen) und die Mortalität an ischämischen Herz- Kreislauferkrankungen (Verstorbene an Krankheiten, die nach ICD-10 mit I20 bis I25 „Ischämische Herzkrankheiten“ kodiert wurden pro 100000 Einwohner/innen) herangezogen. Für den Vergleich zwischen den Bundesländern wurden alle Mortalitätsraten altersstandardisiert (basierend auf der Europäischen Standardbevölkerung nach Eurostat) [22].

\section{Angewandte Analyseverfahren}

Es wurde eine bivariate Korrelation zwischen dem Anteil der Bevölkerung, der die ausdauerorientierten Bewegungsempfehlungen erfüllt (jeweils für die Bewegungsdaten aus dem Österreichischen Bewegungsmonitoring und dem ATHIS) mit den verschiedenen Einflussfaktoren auf Bewegung bzw. mit Gesundheitsindikatoren errechnet. Dabei wurde der Korrelationskoeffizient nach Pearson verwendet. Signifikante Korrelationen $(P<0,05)$ wurden mit * gekennzeichnet.

Bei ausgewählten signifikanten Korrelationen wurde der Zusammenhang zwischen dem Anteil der Bevölkerung, der die ausdauerorientierten Bewegungsempfehlungen erfüllt und den verschiedenen Einflussfaktoren oder Gesundheitsindikatoren mittels Streudiagramm graphisch dargestellt. Die Darstellung erfolgte inklusive Ausgleichsgerade. Außerdem ist in den Graphiken die Formel für die Ausgleichsgerade dargestellt, nach dem Schema: $Y=d+k X$, Wobei k die Maßzahl für die Steigung der Gerade und d den Abstand von der Nulllinie darstellt.

\section{Ergebnisse}

Gemäß österreichischem Bewegungsmonitoring erfüllten 42,6\% der Österreicherinnen und Österreicher ab 15 Jahren die ausdauerorientierten Bewegungsempfehlungen. In den österreichischen Bundesländern variierte dieser Anteil von 38,7 bis 50,4\%. Gemäß österreichischer Gesundheitsbefragung 2014 erfüllten 50,1 \% der Österreicherinnen und Österreicher ab 15 Jahren die ausdauerorientierten Bewegungsempfehlungen. Hierbei variierte der Anteil von 44,9 bis $60,2 \%$.

Weniger als die Hälfte der Befragten (42,0\%) konnten das empfohlene Mindestausmaß der ausdauerorientierten Bewegungsempfehlungen korrekt benennen. Deutlich mehr der Befragten (65,8\%) wussten über die nötige Intensität Bescheid. Gut die Hälfte (57,0\%) meinten, dass der Stellenwert von Bewegung in der Gesellschaft entweder „sehr hoch“ oder „hoch“ sei. In Bezug auf bewegungsförderliche Umwelt wurde in der Wahrnehmung der Österreicherinnen und Österreicher die Möglichkeit zu Fuß zu gehen am besten bewertet (94,3\% bewerteten diese als gut), gefolgt von der Möglichkeit Rad zu fahren (von 90,9\% als gut bewertet) und der Möglichkeit anders körperlich aktiv zu sein, z. B. zu laufen (von 87,6\% als gut bewertet). 83,8\% gaben die Erreichbarkeit von Parks und Grünflächen als gut an, 58,9\% die von Sportvereinen und $55,0 \%$ die Erreichbarkeit von Sportmöglichkeiten ohne Vereine.

In Österreich gab es bezogen auf 100000 Einwohner im Schnitt 4,82 Sportvereine mit zertifizierten gesundheitsfördernden Angeboten für Erwachsene, und in diesen wurden im Schnitt 23,1 zerti- 
fizierte Gesundheitsangebote angeboten. Diesem Angebot standen 12,8 kommerzielle Fitnessbetriebe pro 100000 Einwohner/ innen gegenüber. In Österreich gab es im Schnitt 764 Fahrräder pro 1000 Einwohner und ein Radwegenetz von 163 Metern pro km². Außerdem gab es im Jahr der Erhebung 8,12 Laufveranstaltungen pro 100000 Einwohner.

Die altersstandardisierte Gesamtmortalität betrug in Österreich 1003,5 / 100000 Einwohner/innen, die Mortalität an Herz- Kreislauferkrankungen 434,1 und die an ischämischen Herz- Kreislauferkrankungen 181,1 / 100000 Einwohner/innen. Die Prävalenz chronischer Krankheiten betrug 36,0\%, die von Adipositas 14,3\%, die von Hypertonie $21,1 \%$ und die von Diabetes mellitus $4,9 \%$. $78,6 \%$ der Österreicherinnen und Österreicher beurteilten ihre Gesundheit als „sehr gut“ oder „gut“.

\section{Korrelationen}

Wie in > Tab. 1 dargestellt, gab es eine signifikante positive Korrelation zwischen dem Anteil der Bevölkerung in den Bundesländern, der die Empfehlungen für ausdauerorientierte Bewegung erfüllte und dem Anteil der Bevölkerung, der die richtige Antwort zum Umfang der ausdauerorientierten Bewegungsempfehlungen wusste. Weiter korrelierte der Anteil der Bevölkerung, der die Empfehlungen für ausdauerorientierte Bewegung erfüllte, positiv mit dem Anteil jener die die Erreichbarkeit von Parks und Grünräumen, bzw. die Erreichbarkeit von Angeboten im organisierten Sport bzw. die Möglichkeiten anderwärtig körperlich aktiv zu sein als „sehr gut“ oder als „gut“ bewertete. Zusätzlich korrelierte der Anteil der Bevölkerung, der die Empfehlungen für ausdauerorientierte Bewegung erfüllte positiv mit der Anzahl an Sportvereinen mit gesundheitsförderlichem Angebot sowie der Anzahl zertifizierter gesundheitsförderlicher Angebote in Sportvereinen. In > Abb. 2 sind ausgewählte Zusammenhänge graphisch dargestellt.

Gemäß > Tab. 2 korrelierte der Anteil der Bevölkerung in den Bundesländern, der die Empfehlungen für ausdauerorientierte Bewegung erfüllte, positiv mit dem Anteil der Personen, die ihren Gesundheitszustand als „sehr gut“ oder „gut“ einschätzten und mit der Prävalenz chronischer Krankheiten sowie der Prävalenz von Adipositas negativ. Außerdem korrelierte der Anteil der Bevölkerung, der die Empfehlungen für ausdauerorientierte Bewegung erfüllte, negativ mit der altersstandardisierten Gesamtmortalität, der kardiovaskulären Mortalität und der Mortalität an ischämischen HerzKreislauferkrankungen. In > Abb. $\mathbf{3}$ sind ausgewählte Zusammenhänge grafisch dargestellt.

\section{Diskussion}

Die Ergebnisse zeigen eine starke signifikante positive Korrelation zwischen dem Wissen bezüglich des ausdauerorientierten Bewegungsumfangs, Parametern der gebauten Umwelt und ihrer wahrgenommenen Bewegungsförderlichkeit, sowie manchen (nicht jedoch allen) erhobenen Parametern der bewegungsförderlichen Infrastruktur in den österreichischen Bundesländern und dem

- Tab. 1 Korrelation zwischen dem Anteil der Personen, der die ausdauerorientierten Bewegungsempfehlungen erfüllt, und verschiedenen Einflussfaktoren auf Ebene der österreichischen Bundesländer.

\begin{tabular}{|c|c|c|}
\hline & \multicolumn{2}{|c|}{ Erfüllen der ausdauerorientierten Bewegungsempfehlungen } \\
\hline & $\begin{array}{l}\text { Österreichisches Bewe- } \\
\text { gungsmonitoring } 2017\end{array}$ & $\begin{array}{l}\text { Österreichische Gesund- } \\
\text { heitsbefragung } 2014\end{array}$ \\
\hline $\begin{array}{l}\text { Richtige Antwort auf die Frage „Was meinen Sie, wie anstrengend muss Bewegung } \\
\text { sein, damit man davon gesundheitlich profitiert?“ }\end{array}$ & 0,516 & 0,207 \\
\hline $\begin{array}{l}\text { Richtige Antwort auf die Frage „Und was meinen Sie, wie groß muss der zeitliche } \\
\text { Umfang der Bewegung pro Woche zumindest sein, damit man gesundheitlich davon } \\
\text { profitiert?“ }\end{array}$ & $0,913 * *$ & 0,420 \\
\hline $\begin{array}{l}\text { „Sehr hoch“ oder „eher hoch“ auf „Was meinen Sie, wie hoch ist der Stellenwert in } \\
\text { unserer Gesellschaft sich regelmäßig zu bewegen? }\end{array}$ & $-0,313$ & 0,227 \\
\hline „Sehr gut“ oder „gut“ auf Beurteilung der „Erreichbarkeit von Parks und Grünräumen“ & $0,839 * *$ & 0,610 \\
\hline $\begin{array}{l}\text { "Sehr gut" oder "gut“" auf Beurteilung der „Erreichbarkeit von organisierten } \\
\text { Sportangeboten (z. B. Sportverein)“ }\end{array}$ & 0,405 & $0,845^{* *}$ \\
\hline $\begin{array}{l}\text { "Sehr gut“ oder „gut“ auf Beurteilung der „Erreichbarkeit von organisierten } \\
\text { Sportanlagen außerhalb von Sportvereinen“ }\end{array}$ & 0,171 & 0,619 \\
\hline „Sehr gut" oder „gut" auf Beurteilung der „Möglichkeiten, zu Fuß zu gehen" & 0,464 & 0,496 \\
\hline „Sehr gut“" oder vgut“" auf Beurteilung der „Möglichkeiten, Rad zu fahren“ & 0,385 & 0,432 \\
\hline $\begin{array}{l}\text { "Sehr gut“ oder „Gut“ auf Beurteilung der „Möglichkeiten, auf andere Weise } \\
\text { körperlich aktiv zu sein (z. B. laufen)“ }\end{array}$ & 0,540 & $0,721^{*}$ \\
\hline $\begin{array}{l}\text { Vereine mit Angeboten, wo beide Kategorien „Erwachsene und Senioren“ für } \\
\text { mindestens ein Angebot angekreuzt sind / } 100000 \mathrm{EW}\end{array}$ & $0,760^{*}$ & 0,260 \\
\hline $\begin{array}{l}\text { Anzahl zertifizierte Angebote wobei Kategorien Erwachsene und Senioren gleichzeitig } \\
\text { angekreuzt sind / } 100000 \mathrm{EW}\end{array}$ & $0,870 * *$ & 0,147 \\
\hline Fitnessbetriebe (Fitnessstudios, Fitnesscenter) / $100000 \mathrm{EW}$ & 0,516 & 0,170 \\
\hline Raddichte (Fahrradbesitz pro $1000 \mathrm{EW)}$ & $-0,183$ & 0,506 \\
\hline Radwegdichte (Radwegenetzlänge pro Fläche in m) & $-0,201$ & $-0,478$ \\
\hline Laufveranstaltungen pro Jahr / $100000 \mathrm{EW}$ & 0,177 & $-0,302$ \\
\hline
\end{tabular}




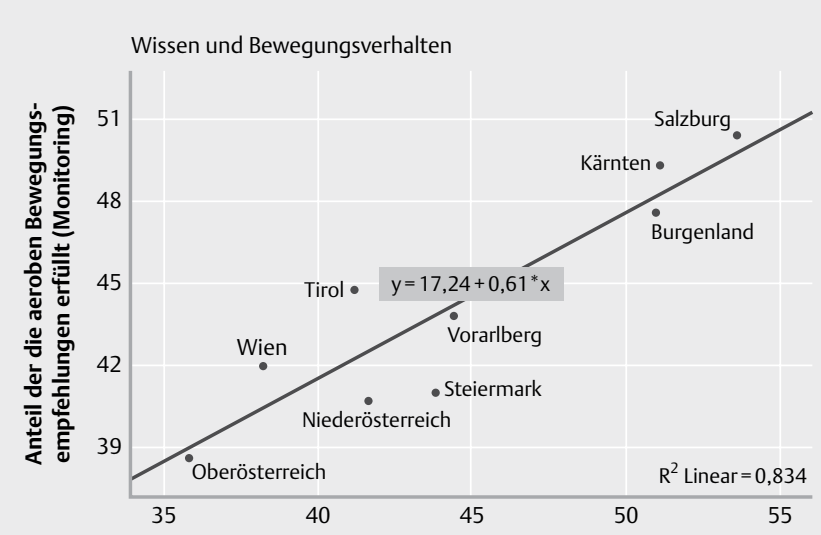

Richtige Antwort auf „Und was meinen Sie, wie groß muss der zeitliche Umfang der Bewegung pro Woche zumindest sein, damit man gesundheitlich davon profitiert?“

bewegungförderliches Umfeld und Bewegungsverhalten

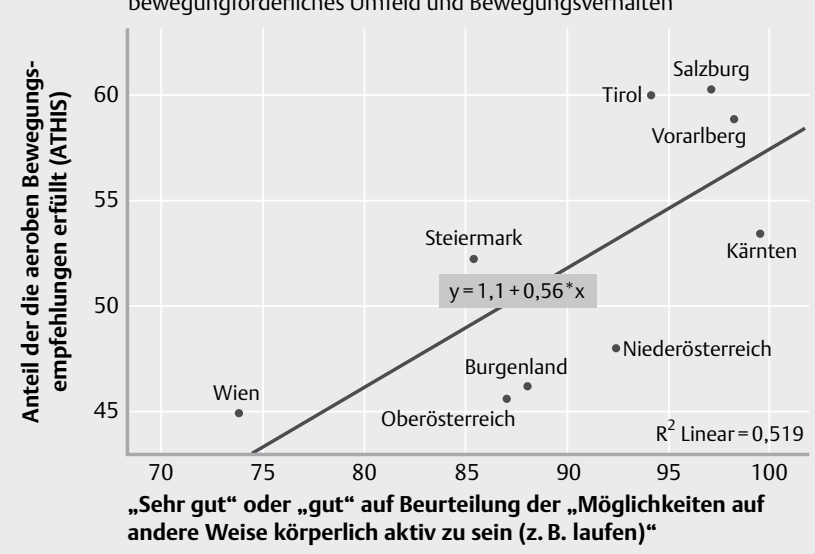

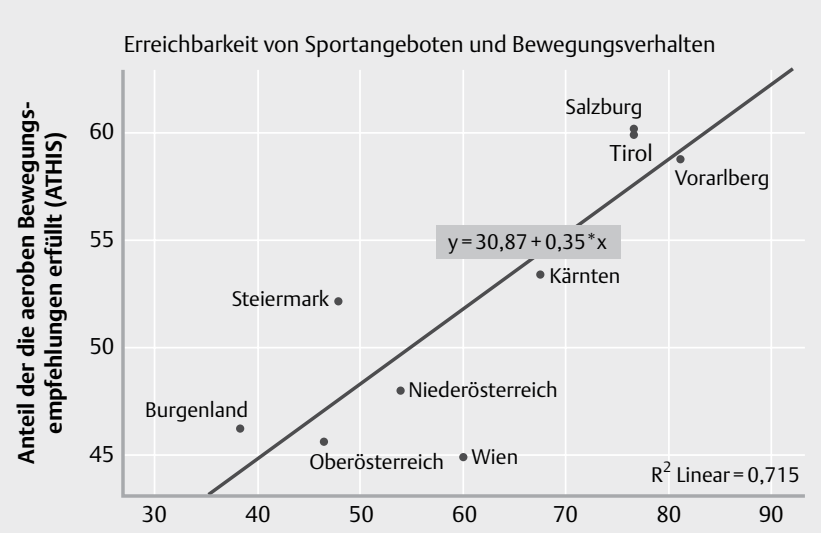

„Sehr gut“ oder „gut“" auf Beurteilung der „Erreichbarkeit von organisierten Sportangeboten (z. B. Sportverein)“

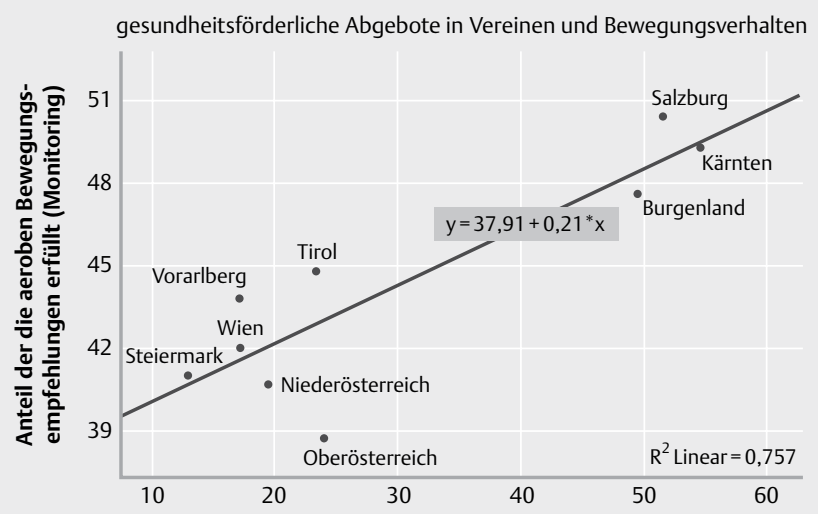

Anzahl zertifizierte Angebote wo beide Kategorien Erwachsene und Senioren gleichzeitig angekreuzt sind/100000 EW

Abb. 2 Zusammenhang zwischen Bewegungsdeterminanten und dem Bewegungsverhalten.

- Tab. 2 Korrelation zwischen dem Anteil der Personen, der die ausdauerorientierten Bewegungsempfehlungen erfüllt, und dem Gesundheitszustand auf Ebene der österreichischen Bundesländer.

\begin{tabular}{|l|l|l|}
\hline & \multicolumn{2}{l}{ Erfüllen der ausdauerorientierten Bewegungsempfehlungen } \\
\cline { 2 - 3 } & $\begin{array}{l}\text { Österreichisches Bewe- } \\
\text { gungsmonitoring 2017 }\end{array}$ & $\begin{array}{l}\text { Österreichische Gesund- } \\
\text { heitsbefragung 2014 }\end{array}$ \\
\hline Subjektiv wahrgenommener Gesundheitszustand „Sehr gut“ oder „gut“ & 0,147 & $0,787^{*}$ \\
\hline Prävalenz chronischer Krankheiten & $-0,016$ & $-0,681^{*}$ \\
\hline Prävalenz Adipositas & $-0,361$ & $-0,828^{* *}$ \\
\hline Prävalenz Hypertonie & 0,023 & $-0,627$ \\
\hline Prävalenz Diabetes mellitus & 0,406 & $-0,453$ \\
\hline Altersstandardisierte Gesamtmortalität auf 100 000 der Bevölkerung & $-0,410$ & $-0,945^{* *}$ \\
\hline $\begin{array}{l}\text { Altersstandardisierte kardiovaskuläre Mortalität auf 100 000 der Bevölkerung } \\
\text { (alle I Diagnosen nach ICD-10) }\end{array}$ & $-0,409$ & $-0,955^{* *}$ \\
\hline $\begin{array}{l}\text { Altersstandardisierte Mortalität an ischämischen Herz-Kreislauferkrankungen auf } \\
\text { 100 000 der Bevölkerung (Diagnosen I20-I25 nach ICD-10) }\end{array}$ & $-0,414$ & $-0,762^{*}$ \\
\hline${ }^{*}$ P<0,05; ${ }^{*}$ P<0,001; EW = Einwohnerinnen und Einwohner. & \\
\hline
\end{tabular}

Bewegungsverhalten in den Bundesländern. Zudem zeigte sich eine starke und signifikant positive Korrelation zwischen dem Bewegungsverhalten in den Bundesländern und der subjektiv wahrgenommenen Gesundheit, genauso wie mit objektiv gemessenen Gesundheitsindikatoren. Obwohl eine Korrelationsstudie keine Rückschlüsse in Bezug auf die Richtung (beeinflusst der eine Fak- tor den anderen oder umgekehrt) oder die Kausalität des Zusammenhanges zulässt, kann (basierend auf aus der Literatur abgeleiteten Hypothesen - dargestellt in $>$ Abb. 1) davon ausgegangen werden, dass das Wissen um die Bewegungsempfehlungen, bewegungsförderliche Kultur und bewegungsförderliche Umgebung in den einzelnen Bundesländern das Bewegungsverhalten beeinflus- 

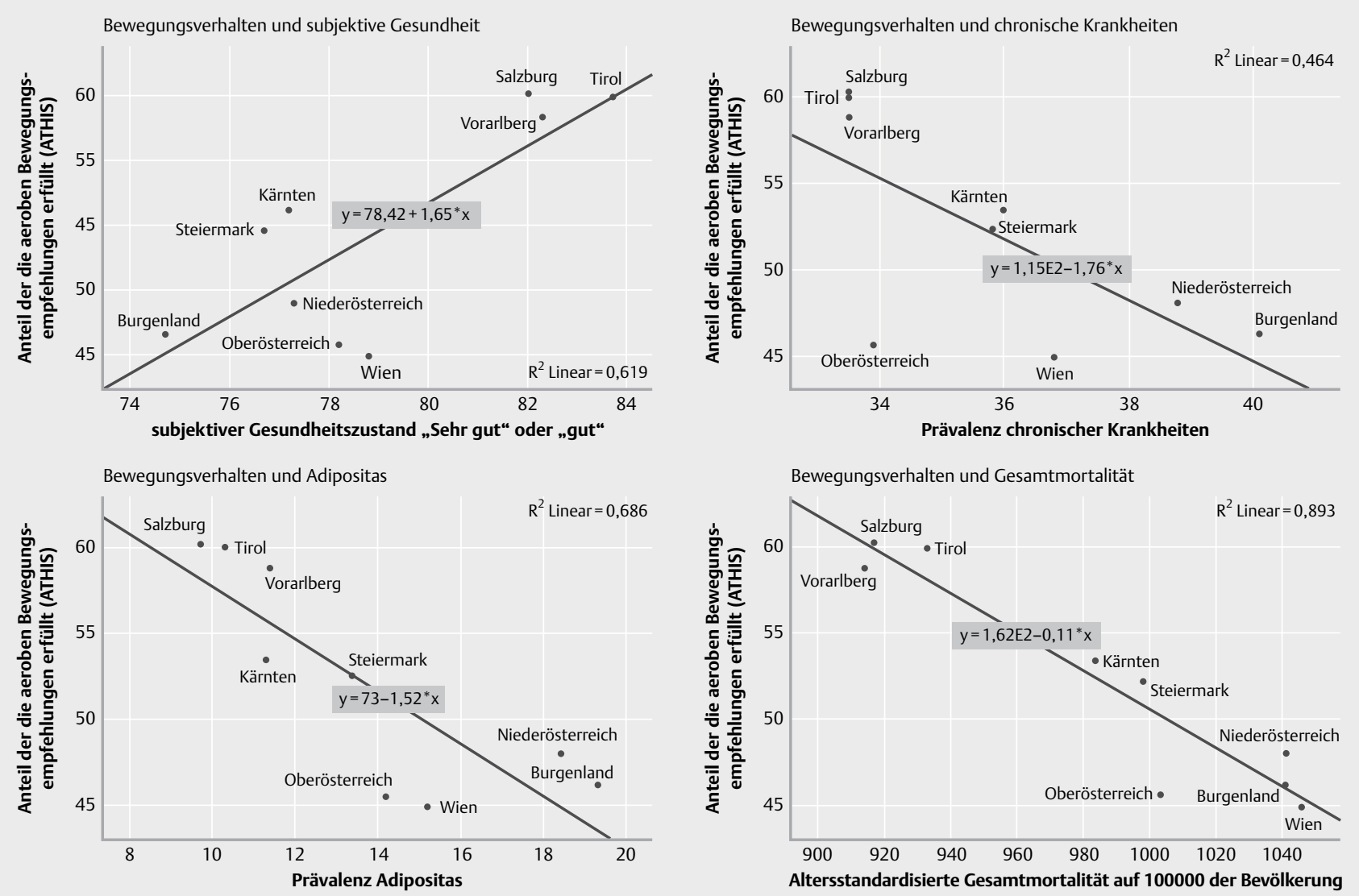

Abb. 3 Zusammenhang zwischen Bewegungsverhalten und Gesundheit.

sen, und dass das Bewegungsverhalten zum Gesundheitszustand der erwachsenen Bevölkerung in den Bundesländern beiträgt.

Ein Zusammenhang zwischen der gebauten und der sozialen Umwelt mit dem Bewegungsverhalten in einer österreichischen Region wurde schon in einer früheren Studie gezeigt [25]. Zudem konnte auch gezeigt werden, dass es einen starken Zusammenhang zwischen dem Bewegungsverhalten in österreichischen Regionen mit dem Gesundheitszustand in diesen Regionen gibt [26].

Viele der dargestellten Assoziationen sind wichtig, um daraus politische Maßnahmen ableiten zu können. So ist der klare Zusammenhang zwischen Wissen um die Bewegungsempfehlungen und dem Bewegungsverhalten ein klarer Auftrag, die Bewegungsempfehlungen auch entsprechend zu kommunizieren. Beispielsweise müssen Multiplikatorinnen und Multiplikatoren die Bewegungsempfehlungen profund kennen und die Kompetenz haben, diese in geeigneter Form zu vermitteln. Aktuell ist der Anteil der österreichischen Bevölkerung, der die ausdauerorientierten Bewegungsempfehlungen kennt, noch relativ gering, wie die hier präsentierten Daten zeigen.

Der hier dargestellte Zusammenhang zwischen der subjektiv wahrgenommen Erreichbarkeit von Angeboten für Bewegung sowie der Zusammenhang zwischen Sportvereinen inklusive deren gesundheitsförderlichen Angeboten, mit dem Bewegungsausmaß zeigen deutlich, dass die Anstrengungen, das Bewegungsverhalten günstig zu beeinflussen, Wirkung zeigen. Damit liefern die Ergeb- nisse eine wichtige Argumentation in Richtung Entscheidungsträgerinnen und Entscheidungsträger, wenn es darum geht, die Umwelt für mehr Bewegung zu gestalten bzw. Sportvereine zu fördern.

Auch der klare Zusammenhang zwischen dem Bewegungsverhalten und der Gesundheit ist für politische Entscheidungen enorm wichtig. Hier konnte ein klarer Zusammenhang zwischen dem Bewegungsverhalten und der subjektiv beurteilten Gesundheit der Prävalenz chronischer Erkrankungen und - als „very hard fact“ mit der Gesamtmortalität sowie der krankheitsspezifischen Mortalität gezeigt werden. Dies ist deshalb wichtig, um zu beweisen, dass die Gesundheit eines Bundeslandes nicht ausschließlich von Entscheidungen im politischen Gesundheitsressort, sondern auch in einem großen Maß in anderen politischen Handlungsfeldern, wie im Sport, in der Bildung oder in der Infrastruktur- und Landschaftsplanung, generiert wird.

Eine Stärke der Studie ist, dass der Analyse valide Datensätze zugrunde liegen, mit denen das Bewegungsverhalten mit standardisierten und validen Instrumenten erhoben wurde, und die auch über die nötigen Stichprobenumfänge verfügen, um auf Bundesländerebene Aussagen treffen zu können. Als weitere Stärke ist anzuführen, dass der Analyse eine umfangreiche Recherche zugrunde liegt, welche Datensätze grundsätzlich in Österreich zur Verfügung stehen um Determinanten des Bewegungsverhaltens, das Bewegungsverhalten selbst und die Gesundheit in den Bundesändern darstellen zu können. Sehr selten werden diese verschiedenen 
Datenquellen wie hier zusammengeführt und einer gemeinsamen Analyse unterzogen. Zu den Limitierungen dieser Studie ist anzumerken, dass die hier dargestellten Korrelationen ausschließlich mit den aggregierten Daten auf Bundesländerebene erfolgten und somit gibt es nur neun Beobachtungseinheiten (die 9 Bundesländer). Bei dieser kleinen Zahl an Beobachtungseinheiten kann es sein, dass eine starke Korrelation zwischen 2 Merkmalen vorhanden ist, diese aber statistisch nicht signifikant ist. Auch die einzelnen Datenquellen haben ihre Einschränkungen. Beim Bewegungsmonitoring 2017 ist anzumerken, dass eine Antwortrate nicht übermittelt wurde. Somit ist auch nicht einzuschätzen, ob und in welcher Höhe es mögliche Verzerrungen gibt. Als weitere Limitierung beider Befragungsdatensätze, dem Bewegungsmonitoring 2017 und der Gesundheitsbefragung 2014 ist anzuführen, dass alle Variablen selbstberichtet sind und dadurch auch der Verzerrung unterliegen, sodass möglicherweise sozial erwünschte Antworten gegeben wurden. Hierbei wird bspw. bei Befragungen mit dem GPAQ die selbstberichtete wöchentliche Bewegung überschätzt [11]. Trotz intensivster Recherchen ist es nicht gelungen, etwa Daten zum Budget für Sportförderung, für Sport gewidmete Flächen, Anzahl von Sportstätten, Anzahl an Gesundheits- oder Breitensportveranstaltungen, oder Bewegungsinitiativen in den einzelnen Bundesländern zu erhalten, um Zusammenhänge mi dem Bewegungsverhalten und mit der Gesundheit zu untersuchen.

\section{FAZIT FÜR DIE PRAXIS}

Die Ergebnisse der Analysen zeigen, dass der Anteil an Personen, der die ausdauerorientierten Bewegungsempfehlungen erfüllt, in den Bundesländern sehr unterschiedlich und in allen verbesserungsbedürftig, ist. Diesbezüglich, aber auch in Bezug auf das Wissen um die Bewegungsempfehlungen, die bewegungsförderliche Kultur und Infrastruktur gibt es ein großes Verbesserungspotenzial. Viele Einflussfaktoren auf das Bewegungsverhalten einer Bevölkerung liegen außerhalb des politischen Sportresorts (z. B. im Bildungsbereich, im Verkehrsbereich, oder im Bereich der Raumplanung). Eine Vernetzung mit diesen Resorts ist unumgänglich und Synergien sollten gesucht und forciert werden. Es gibt einen sehr starken Zusammenhang zwischen der Erfüllung der ausdauerorientierten Bewegungsempfehlungen und dem Gesundheitszustand. Dies reicht von der subjektiven Gesundheitseinschätzung über das Vorhandensein chronischer Erkrankungen bis hin zu Indikatoren der Mortalität. Der Gesundheitssektor profitiert von einem dichten und attraktiven Sportangebot. Der Sportsektor wiederum kann zusätzlich Erwachsene ansprechen, indem neben sozialem Erleben und der Verbesserung der Fitness auch die Gesundheit als Motiv beworben werden kann.

\section{Danksagung}

Die Bundesländeranalyse und diese open access Publikation wurden dankenswerter Weise vom österreichischen Bundesministerium für Kunst, Kultur, öffentlichen Dienst und Sport, der Gesundheit Österreich $\mathrm{GmbH}$, Geschäftsbereich Fonds Gesundes Öster- reich, und der Österreichischen Gesellschaft für Public Health unterstützt.

\section{Interessenskonflikt}

Die Autorinnen/Autoren geben an, dass kein Interessenskonflikt besteht.

\section{Literatur}

[1] US Department of Health and Human Services Physical Activity Guidelines for Americans. $2^{\text {nd }}$ ed. Washington, D.C: US Department of Health and Human Services; 2018. Abgerufen am 24. 2020 von https://health.gov/sites/default/files/2019-09/Physical_Activity_ Guidelines_2nd_edition.pdf

[2] Mayer S, Felder-Puig R, Gollner E et al. Bewegungsverhalten, Kosten mangelnder körperlicher Aktivität und Bewegungsförderung in Österreich. Das Gesundheitswesen 2020; 82 (Suppl. 3): S196-S206

[3] Miko HC, Zillmann N, Ring-Dimitriou S et al. Auswirkungen von Bewegung auf die Gesundheit. Das Gesundheitswesen 2020; 82 (Suppl. 3): S184-S195

[4] Titze S, Lackinger C, Fessl C et al. Österreichische Bewegungsempfehlungen für Erwachsene, inklusive ältere Erwachsene und Menschen mit chronischen Erkrankungen. Das Gesundheitswesen 2020; 82 (Suppl. 3): S170-S176

[5] Dahlgren G, Whitehead M. Policies and strategies to promote social equity in health.Stockholm: Institute for future studies 1991; 27: 4-41

[6] Titze S, Dorner TE, Ropin K et al. Editorial: Warum Österreichische Bewegungsempfehlungen? Das Gesundheitswesen 2020; in Druck

[7] Bachner F, Bobek J, Habimama K et al. Das österreichische Gesundheitssystem - Akteure, Daten, Analysen. Gesundheitssysteme im Wandel 2019; 20: 1-288

[8] Titze S, Halbwachs C, Gollner E et al. Bundesministerium für Landesverteidigung und Sport., Hrsg. Durchführung eines Bewegungsmonitoring-Systems in Österreich. Wien: Eigenverlag; 2016

[9] BMLV (Bundesministerium für Landesverteidigung und Sport). Bewegungs-Monitoring Bevölkerungsbefragung 2017. Abgerufen am 24. April 2020, von https://www.bmkoes.gv.at/sport/breitensport/ breiten-gesundheitssport/Bewegungsmonitoring.htmlbzw https:// www.bmkoes.gv.at/dam/jcr:ff29381b-f862-4304-b29e-8bab0350a9cf/Studienbericht\%20Bewegungsmonitoring\%202017.pdf

[10] Statistik Austria. Die Österreichische Gesundheitsbefragung 2014 (ATHIS). Wien: Statistik Austria, 2015. Abgerufen am 24. April 2020, von https://www.statistik.at/web_de/services/publikationen/4/index.html?i ncludePage $=$ detailedView\&sectionName $=$ Gesundheit\&publd $=714$

[11] Chu $\mathrm{AH}, \mathrm{Ng} \mathrm{SH}$, Koh D et al. Reliability and validity of the self-and interviewer-administered versions of the Global Physical Activity Questionnaire (GPAQ). PLoS One 2015; 10: e0136944

[12] Cleland CL, Hunter RF, Kee F et al. Validity of the global physical activity questionnaire (GPAQ) in assessing levels and change in moderate-vigorous physical activity and sedentary behaviour. BMC public health 2014; 14: 1255

[13] Stamm H, Wiegand D, Lamprecht M. Monitoring-System Ernährung und Bewegung. Indikatoren-sammlung zum NPEB 2008-2012. Indikator 2.7: Stillen. Bern: Bundesamt für Gesundheit; 2015

[14] Österreichisches Jugendportal. Bewegung und Sport. 2013. Abgerufen am 24. April 2020, von https://www.jugendportal.at/bewegung-undsport

[15] Eurobarometer S. 412. Sport and physical activity. Brussels: European Commission; Directorate General for Education and Culture. 2014 
[16] Bödeker M, Bucksch J, Fuhrmann H. Bewegungsfreundlichkeit von Wohnumgebungen messen. Entwicklung und Einführung der Deutschsprachigen „Neighborhood Environment Walkability Scale“. Prävention und Gesundheitsförderung 2012; 7: 220-226

[17] Eurostat European Commission European Health Interview Survey (EHIS wave 2) Methodological manual. Publications Office of the European Union. 2013. Abgerufen am 24. 2020; von https://ec. europa.eu/eurostat/documents/3859598/5926729/KS-RA-13-018-EN. PDF/26c7ea80-01d8-420e-bdc6-e9d5f6578e7c

[18] Aromaa A, Koponen P, Tafforeau J et al. Evaluation of health interview surveys and health examination surveys in the European Union. European Journal of Public Health 2003; 13 (suppl_3): 67-72

[19] Finger JD, Jonas D, Gisle L et al. Development of the European health interview survey-physical activity questionnaire (EHIS-PAQ) to monitor physical activity in the European Union. Arch Public Health 2015; 73: 59

[20] Baumeister SE, Sebastian E, Kohler S et al. Physical activity surveillance in the European Union: reliability and validity of the European health interview survey-physical activity questionnaire (EHIS-PAQ). Int J Behav Nutr Phys Act 2016; 13: 61
[21] Fit Sport Austria. Abgerufen am 24. April 2020, von https://www. fitsportaustria.at $/$ main.asp?VID $=1 \&$ kat $1=108 \&$ kat $2=715$

[22] Statistik Österreich Statistik Austria „Todesursachen 2015“. In: Jahrbuch der Gesundheitsstatistik 2015. Bundesanstalt Statistik Österreich. Wien: 2017

[23] WKO (Wirtschaftskammer Österreich) Statistik Österreich, Freizeit und Sportbetriebe: Branchendaten. Wien: WKÖ, Abteilung für Statistik; 2019

[24] BMVIT (Bundesministerium für Verkehr, Innnovation und Technologie), Hrsg. Radverkehr in Zahlen. Daten, Fakten und Stimmungen. 2. Auflage Wien: 2013

[25] Eichinger M, Titze S, Haditsch B et al. How are physical activity behaviors and cardiovascular risk factors associated with characteristics of the built and social residential environment? PLoS One 2015; 10: e0126010

[26] Stein KV, Rieder A, Dorner TE. East-West gradient in cardio-vascular mortality in Austria: how much can we explain by following the pattern of risk factors? Int J Health Geogr 2011; 10: 59 